HUB-EP-97/80

\title{
Hadronization in Particle Physics
}

\author{
D.Ebert* \\ Institut für Physik, Humboldt-Universität zu Berlin, \\ Invalidenstrasse 110, D-10115, Berlin, Germany
}

\begin{abstract}
The method of path integral hadronization is applied to a local quarkdiquark toy model in order to derive an effective chiral meson-baryon Lagrangian. Further generalizations to models including both scalar and axial-vector diquarks as well as nonlocal interactions are discussed.
\end{abstract}

\section{Introduction}

In the first lecture (referred to as $I$ in the following) I have shown that QCDmotivated effective quark models of the NJL type can be reformulated as effective theories given in terms of composite bosonic objects, mesons. An analogous treatment of baryons as relativistic bound systems leads us in the case $N_{\mathrm{c}}=3$ to the concept of diquarks. Diquarks as effective degrees of freedom have been introduced both in two-dimensional QCD [1] and in four-dimensional QCDtype models [2]. By applying path integral methods to an NJL-type model with 2-body $(q \tilde{q})$ and $(q q)$ forces it was, in particular, possible to derive Faddeev equations determining the spectrum of composite baryons [3-5]. It is a further challenge to derive the well known effective chiral meson-baryon Lagrangians $[6,7]$, which reproduce low energy characteristica of hadron physics directly by the path integral hadronization method.

In this second lecture I will demonstrate how this can be done by choosing a simple quark-diquark toy model containing a local interaction of elementary scalar diquarks with quarks. Finally, possible generalizations to models containing scalar and axial-vector diquarks taken as elementary fields [8] or composite fields and including nonlocal interactions mediated by quark exchange [3-5,9] are discussed.

\section{Hadronization of a Quark-Diquark Toy Model}

Let us consider a chiral-invariant Lagrangian containing the semi-bosonized meson-quark Lagrangian $\mathcal{L}_{\text {NJL }}^{\chi \mathrm{M}}$ described in lecture $I$ (see (22)) supplemented by a diquark and an interaction term

\footnotetext{
^ Invited talk given at the Workshop "Field Theoretical Tools in Polymer and Particle Physics", University Wuppertal, June 17-19, 1997
} 


$$
\mathcal{L}^{\chi \mathrm{MD}}=\mathcal{L}_{\mathrm{NJL}}^{\chi \mathrm{M}}+D^{\dagger}\left(-\square-M_{D}^{2}\right) D+\tilde{G}\left(\bar{\chi} D^{\dagger}\right)(D \chi) .
$$

Here $D(x)$ is the field of an (elementary) scalar isoscalar diquark of mass $M_{D}$, and $\tilde{G}$ is a coupling constant.

Analogously to the introduction of collective meson fields into the NJL model (Cf. (4) of $I$ ) let us now introduce collective baryon (nucleon) fields $B$ by using the identity

$$
\mathrm{e}^{\mathrm{i} \int \mathrm{d}^{4} x \tilde{G}\left(\bar{\chi} D^{\dagger}\right)(D \chi)}=\mathcal{N}^{\prime} \int \mathcal{D} B \mathcal{D} \bar{B} \mathrm{e}^{\mathrm{i} \int \mathrm{d}^{4} x\left(-\frac{1}{G} \bar{B} B-\bar{\chi} D^{\dagger} B-\bar{B} D \chi\right)} .
$$

The "hadronization" of the generating functional of the Lagrangian (1) will now be performed step by step by integrating over the microscopic quark and diquark fields. We obtain

$$
\begin{gathered}
\mathcal{Z}=\mathcal{N}_{1} \int \mathcal{D} \mu\left(\tilde{\sigma}, \varphi_{i}, B, D\right) \mathrm{e}^{\mathrm{i} \int \mathrm{d}^{4} x\left[-\mathrm{i} \operatorname{tr} \ln S_{\chi}^{-1}-\frac{1}{\bar{G}} \bar{B} B\right]} \times \\
\mathrm{e}^{\mathrm{i} \iint \mathrm{d}^{4} x \mathrm{~d}^{4} y\left[D^{\dagger}(x)\left(\Delta^{-1}-\bar{B} S_{\chi} B\right)_{(x, y)} D(y)\right]}, \\
\mathcal{Z}=\mathcal{N}_{2} \int \mathcal{D} \mu\left(\tilde{\sigma}, \varphi_{i}, B\right) \exp \left\{\mathrm { i } \int \mathrm { d } ^ { 4 } x \left[-\mathrm{itr} \ln S_{\chi}^{-1}-\frac{1}{\tilde{G}} \bar{B} B+\right.\right. \\
\left.\left.\operatorname{iln}\left(1-\bar{B} S_{\chi} \Delta B\right)_{(x, x)}\right]\right\},
\end{gathered}
$$

where the trace tr runs over Dirac, isospin, and colour indices, $\mathcal{D} \mu$ denotes the integration measure of fields, $\Delta^{-1}=-\square-M_{D}^{2}$ is the inverse diquark propagator, and $S_{\chi}$ is the quark propagator defined by (Cf. (28) of $I$ )

$$
S_{\chi}^{-1}=\mathrm{i} \hat{D}-m-\tilde{\sigma}-\hat{\mathcal{A}} \gamma_{5}
$$

Expanding now the logarithms in power series at the one-loop level (see Fig.1) and performing a low-momentum expansion of Feynman diagrams (corresponding to a derivative expansion in configuration space), one describes both the generation of kinetic and mass terms of the composite baryon field $B$. This yields the expression

$$
\int \mathrm{d}^{4} x \mathrm{~d}^{4} y \bar{B}(x)\left[-\left(\frac{1}{\tilde{G}}+Z_{1}^{-1} \frac{\boldsymbol{\tau}}{2} \hat{\mathcal{V}}+g_{A} \frac{\boldsymbol{\tau}}{2} \hat{\mathcal{A}} \gamma_{5}\right) \delta^{4}(x-y)-\Sigma(x-y)\right] B(y) .
$$

The vertex renormalization constant $Z_{1}^{-1}$ and the axial coupling $g_{A}$ arise from the low-momentum (low derivative) expansion of the vertex diagrams of Fig.1 b) and c), respectively. The nucleon self-energy $\Sigma$ has in momentum space the decomposition $\Sigma(p)=\hat{p} \Sigma_{\mathrm{V}}\left(p^{2}\right)+\Sigma_{\mathrm{S}}\left(p^{2}\right)$. Its low momentum expansion generates a kinetic term $\left(\sim Z^{-1} \hat{p}\right)$ and, together with the constant term $-1 / \tilde{G}$, a mass $M_{B}$ given by the equation 


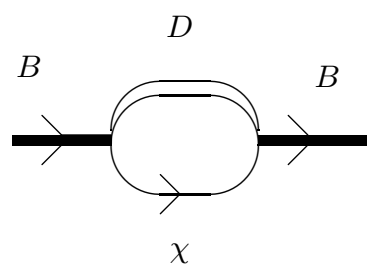

(a)

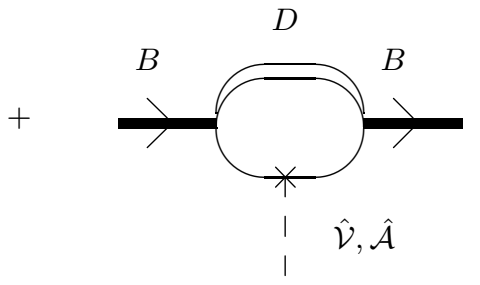

$(\mathrm{b}, \mathrm{c})$

Fig. 1. Baryon self-energy diagram $\Sigma$ (a) and vertex diagrams (b,c) arising from the loop expansion of the logarithms in Eq. (4).

$$
\frac{1}{\tilde{G}}+M_{B} \Sigma_{\mathrm{V}}\left(M_{B}^{2}\right)+\Sigma_{\mathrm{S}}\left(M_{B}^{2}\right)=0 .
$$

In terms of renormalized fields defined by $B=Z^{\frac{1}{2}} B_{\mathrm{r}}$, where $Z$ is the wave function renormalization constant satisfying the Ward identity $Z=Z_{1}$, we obtain from (6) the effective chiral meson-baryon Lagrangian [10]

$$
\mathcal{L}_{\text {eff. }}^{\mathrm{MBB}}=\bar{B}_{\mathrm{r}}\left(\mathrm{i} \hat{D}-M_{B}\right) B_{\mathrm{r}}-g_{A}^{\mathrm{r}} \bar{B}_{\mathrm{r}} \gamma_{\mu} \gamma_{5} \frac{\tau_{i}}{2} B_{\mathrm{r}} \mathcal{A}_{i}^{\mu},
$$

with $\hat{D}=\hat{\partial}+\mathrm{i} \hat{\mathcal{V}}$ and $g_{A}^{\mathrm{r}}=Z g_{A}$ being the renormalized axial coupling constant. Note that expression (8) completely coincides in structure with the famous phenomenological chiral Lagrangians introduced at the end of the Sixties when considering nonlinear realizations of chiral symmetry $[6,7]$. However, in our case these Lagrangians are not obtained on the basis of symmetry arguments alone, but derived from an underlying microscopic quark-diquark picture which allows us to estimate masses and coupling constants of composite hadrons. Note that, due to $\mathcal{A}_{\mu}^{i}=\frac{1}{F_{\pi}} \partial_{\mu} \varphi^{i}+\cdots$, the second term in (8) leads to a derivative coupling of the pion field $\varphi$ with the axial-vector baryon current. In order to get rid of the derivative and to reproduce the standard $\gamma_{5}$ coupling, it is convenient to redefine the baryon field $B_{\mathrm{r}} \rightarrow \tilde{B}$ by

$$
B_{\mathrm{r}}=\mathrm{e}^{-\mathrm{i} g_{A}^{\mathrm{r}} \gamma_{5} \frac{\tau \cdot \varphi}{2 F_{\pi}}} \tilde{B} .
$$

Inserting (9) into (8) and performing a power series expansion in $\varphi$ leads to the expression

$$
\mathcal{L}_{\text {eff. }}^{\mathrm{MB}}=\overline{\tilde{B}}\left(\mathrm{i} \hat{\partial}-M_{B}\right) \tilde{B}+g_{A}^{\mathrm{r}} \frac{M_{B}}{F_{\pi}} \overline{\tilde{B}} \mathrm{i} \gamma_{5} \tau \cdot \varphi \tilde{B}+O\left(\varphi^{2}\right) .
$$

Obviously, we have to identify the factor in front of the interaction term as the pion-nucleon coupling constant $g_{B B \varphi}$,

$$
g_{B B \varphi}=g_{A}^{\mathrm{r}} \frac{M_{B}}{F_{\pi}}
$$


which is nothing else than the Goldberger-Treiman relation of the composite nucleon. Finally, by combining the Lagrangian (8) with the effective chiral meson Lagrangian of the nonlinear $\sigma$ model (Cf. (31) of $I$ ), we obtain the complete meson-baryon Lagrangian

$$
\mathcal{L}_{\text {eff., tot. }}^{\mathrm{MB}}=\mathcal{L}_{\text {nlin. }}^{\sigma}+\mathcal{L}_{\text {eff. }}^{\mathrm{MB}} .
$$

It is further possible to estimate magnetic moments as well as electric and magnetic radii of composite protons and neutrons by introducing electromagnetic interactions into the toy Lagrangian (1). The obtained pattern of predicted low-energy characteristica of nucleons has been shown to describe data at best qualitatively [11]. Obviously, in order to get better agreement with data, it is necessary to consider more realistic, but also more complicated models with nonlocal quark-diquark interactions containing both scalar and axial-vector diquarks [3-5,12].

\section{$3 \quad$ Further Extensions}

\subsection{Heavy Baryons with Scalar and Axial-Vector Diquarks}

The above considerations can be easily generalized to heavy-light baryons $B \sim(Q q q)$, where $Q=c, b$ is a heavy quark and the light quarks $q=u, d, s$ form scalar $(D)$ and axial-vector diquarks $\left(F_{\mu}\right)$ of the flavour group $S U(3)_{\mathrm{F}}$. The quantum numbers of the light diquarks with respect to the spin, flavour, and colour follow here from the decomposition

$$
\begin{gathered}
\text { spin : } \frac{1}{2} \times \frac{1}{2}=0_{\mathrm{a}}+1_{\mathrm{s}} \\
\text { flavour : } 3_{\mathrm{F}} \times 3_{\mathrm{F}}=\overline{3}_{\mathrm{F}, \mathrm{a}}+6_{\mathrm{F}, \mathrm{s}} \\
\text { colour : } 3_{\mathrm{c}} \times 3_{\mathrm{c}}=\overline{3}_{\mathrm{c}, \mathrm{a}}\left(+6_{\mathrm{c}, \mathrm{s}}\right),
\end{gathered}
$$

where the indices s,a refer to symmetry, antisymmetry of the respective wave functions under interchange of quark indices. According to the Pauli principle the fields of the scalar and axial-vector diquarks must then form a flavour (anti)triplet or sextet, respectively: $D_{\overline{3}_{\mathrm{F}}}, F_{6_{\mathrm{F}}}^{\mu}$. The baryons as bound states of diquarks with a quark $Q$ are evidently colourless, since the product representation $\overline{3}_{\mathrm{c}} \times 3_{\mathrm{c}}$ contains the colour singlet 1 . In Ref. [8] we have studied an extended quark-diquark model given by the Lagrangian

$$
\begin{gathered}
\mathcal{L}=\mathcal{L}_{0}+\mathcal{L}_{\text {int. }}, \\
\mathcal{L}_{0}=\bar{q}\left(\mathrm{i} \hat{\partial}-m_{0}\right) q+\bar{Q}_{\mathrm{v}} \mathrm{i} v \cdot \partial Q_{\mathrm{v}}+\operatorname{tr}\left[\partial_{\mu} D^{\dagger} \partial^{\mu} D-M_{D}^{2} D^{\dagger} D\right]
\end{gathered}
$$




$$
\begin{gathered}
-\frac{1}{4} \operatorname{tr} F_{\mu \nu}^{\dagger} F^{\mu \nu}+\operatorname{tr} M_{\mathrm{F}}^{2} F_{\mu}^{\dagger} F^{\mu}, \\
\mathcal{L}_{\text {int. }}=\tilde{G}_{1} \operatorname{tr}\left(\bar{Q}_{v} D^{\dagger}\right)\left(D Q_{v}\right)-\tilde{G}_{2} \operatorname{tr}\left(\bar{Q}_{v} F^{\dagger \mu}\right) P_{\mu \nu}^{\perp}\left(F^{\nu} Q_{v}\right),
\end{gathered}
$$

where $D^{i j}, F_{\mu}^{i j}$ are antisymmetric/symmetric $3 \times 3$ flavour matrices, $Q_{v}$ is a heavy quark spinor of 4-velocity $v_{\mu}$ (using the notation of Heavy Quark Effective Theory [13]), $P_{\mu \nu}^{\perp}=g_{\mu \nu}-v_{\mu} v_{\nu}$ is a transverse projector, and $\tilde{G}_{1}, \tilde{G}_{2}$ are coupling constants.

In order to bilinearize the interaction term (15) one now needs two types of baryon fields

$$
T_{v}\left(\frac{1}{2}^{+}\right) \sim Q_{v} D, \quad S_{v \mu}\left(\frac{1}{2}^{+}, \frac{3}{2}^{+}\right) \sim Q_{v} F_{\mu}
$$

where $S_{v \mu}$ is a superfield of spin-symmetry partners $B_{v}\left(\frac{1}{2}^{+}\right), B_{v \mu}^{*}\left(\frac{3}{2}^{+}\right)$admitting the decomposition

$$
S_{v \mu}=\frac{1}{\sqrt{3}} \gamma_{5}\left(\gamma_{\mu}-v_{\mu}\right) B_{v}+B_{v \mu}^{*} .
$$

The interaction term (15) can then be rewritten as a sum of terms bilinear in the baryon field and a Yukawa interaction term. Integrating successively over the quark fields $q, Q$ and then over the diquark fields $D, F_{\mu}$ leads to determinants containing the baryon fields $T$ and $S$. Finally, by employing again a loop expansion and taking into account only lowest order derivative terms, leads to the free effective baryon Lagrangian of heavy flavour type

$$
\mathcal{L}_{\text {eff. }}^{0}=\operatorname{tr} \bar{T}_{v}\left(\mathrm{i} v \cdot \partial-\Delta M_{\mathrm{T}}\right) T_{v}-\operatorname{tr} \bar{S}_{v \mu}\left(\mathrm{i} v \cdot \partial-\Delta M_{\mathrm{S}}\right) S_{v}^{\mu},
$$

where the mass differences $\Delta M_{\mathrm{T}, \mathrm{S}} \equiv M_{\mathrm{T}, \mathrm{S}}-m_{\mathrm{Q}}$ are calculable. Moreover, taking into account vertex diagrams analogously to those shown in Fig.1 (b,c) leads to

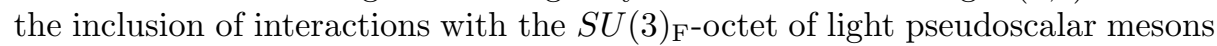
$\varphi_{i}[8]$.

\subsection{Composite Diquarks}

For simplicity, we have considered up to now only models containing diquarks as elementary fields. Clearly, it is desirable to treat diquarks on the same footing as composite mesons as composite particles. This has been done in Ref. [9] by considering an extended NJL model for light $(q=u, d, s)$ and heavy quarks $(Q=c, b)$ containing 2-body interactions of diquark-type $(q q)$ and $(q Q)$

$$
\mathcal{L}_{\mathrm{NJL}}^{\text {int. }}=G_{1}\left(\bar{q}^{\mathrm{c}} \Gamma^{\alpha} q\right)\left(\bar{q} \Gamma^{\alpha} q^{\mathrm{c}}\right)+G_{2}\left(\bar{q}^{\mathrm{c}} \Gamma_{v}^{\alpha} Q_{v}\right)\left(\bar{Q}_{v} \Gamma_{v}^{\alpha} q\right) .
$$

Here $q^{\mathrm{c}}=C \bar{q}^{\mathcal{T}}$ denotes a charged-conjugated quark field, and $\Gamma^{\alpha}, \Gamma_{v}^{\alpha}$ are flavour and Dirac (spin) matrices. The interaction term (18) can again be rewritten in 
terms of a Yukawa coupling of light composite scalar and axial-vector diquark fields $D\left(0^{+}, 1^{+}\right)$or heavy diquarks $D_{v}\left(J^{\mathrm{P}}=0^{+}, 1^{+}\right)$to quarks. Notice that these models lead to a nonlocal quark-diquark interaction mediated by quark exchange shown in Fig.2.

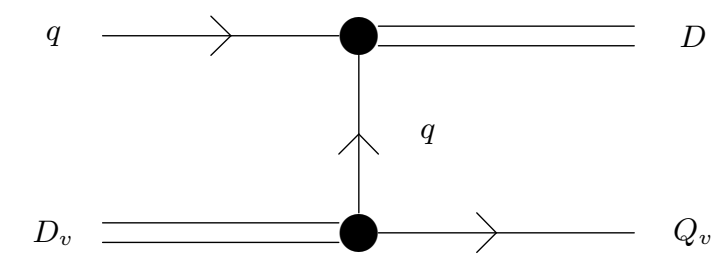

Fig. 2. Quark-exchange diagram leading to a nonlocal quark-diquark interaction with composite diquarks.

As shown in a series of papers [3-5,9], the baryon spectrum can then be found by solving Faddeev-type equations for quark-diquark bound states. Finally, the above method of path integral hadronization can also be applied to investigate 3-body interactions of the type $\mathcal{L} \sim(\bar{q} \bar{q} \bar{q})(q q q)$ [5].

I hope that these few examples are sufficient to show you that path integral hadronization is indeed a powerful nonperturbative method in particle physics.

\section{References}

1. Ebert, D. and Pervushin, V.N.: Teor. Mat. Fiz. 36 (1978), 759; Ebert, D. and Kaschluhn, L.: Nucl. Phys. B355 (1991), 123.

2. Cahill, R.T., Praschifka, J., and Burden, C.J.: Aust. J. Phys. 42 (1989), 147, 161; Kahana, D. and Vogl, U.: Phys. Lett. B244 (1990), 10; Ebert, D., Kaschluhn, L., and Kastelewicz, G.: Phys. Lett. B264 (1991), 420.

3. Cahill, R.T.: Aust. J. Phys. 42 (1989), 171.

4. Reinhardt, H.: Phys. Lett. B244 (1990), 316.

5. Ebert, D. and Kaschluhn, L.: Phys. Lett. B297 (1992), 367.

6. Coleman, S., Wess, I., and Zumino, B.: Phys. Rev. 177 (1969), 2239; ibid. 2247 .

7. Ebert, D. and Volkov, M.K.: Fortschr. Phys. 29 (1981), 35.

8. Ebert, D., Feldmann, T., Kettner, C., and Reinhardt, H.: Z. Phys. C71 (1996) 329.

9. Ebert, D., Feldmann, T., Kettner, C., and Reinhardt, H.: Preprint DESY-96010, to appear in Int. J. Mod. Phys. A. 
10.Ebert, D. and Jurke, T.: Preprint HUB-EP-97/74; hep-ph/9710390 (1997). 11.Jurke, T.: Diploma Thesis, Humboldt University, Berlin, (1997).

12.Keiner, V.: Z. Phys. A354 (1996), 87.

13.Isgur, N. and Wise, M.: Phys. Rev. D41 (1990), 151; Neubert, M.: Phys. Rept. 245 (1994), 259. 